\title{
Structure Factor for Quasicrystals 1D Case
}

\author{
B. KOZAKOWSKI AND J. WOLNY \\ Faculty of Physics and Nuclear Techniques, AGH-UST \\ al. Mickiewicza 30, 30-059 Kraków, Poland
}

(Received May 13, 2003)

\begin{abstract}
Using a statistical approach a simple formula for the structure factor of decorated Fibonacci chain was derived. Although the used method operates in the physical space only, its equivalence to the higher-dimensional analysis was proved. Applications of the analysis to different decorated structures were also discussed.
\end{abstract}

PACS numbers: 61.44.Br, 61.43.-j, 61.10.Dp

\section{Introduction}

After the famous discovery of quasicrystals [1] the question "where are the atoms?" [2] is still open. More accurate measurements have been performed and the new ideas of data analysis have been elaborated: like higher-dimensional Patterson analysis [3, 4], X-ray and neutron diffraction [5], density modification method [6], atomic-resolution annual dark-field scanning transmission electron microscopy (STEM) [7], electron diffraction and STEM [8]. Recently a new statistical approach to aperiodic structures (including quasicrystals) has been formulated and used for different model structures in one and two dimensions [9-11], like: 1D Fibonacci chain or sinusoidal modulated structures, 2D Penrose tiling or random structures. Similar approach has been also formulated in higher-dimensional reciprocal space [12]. The goal of the statistical approach is to derive rather simple formulae (in physical space) which can be easily applied to the structure refinement from the obtained diffraction pattern. In this paper the main idea of the used approach is presented and tested on the simple 1D model of the Fibonacci chain. This idea can then be easily extended to more complicated 2D and 3D structures, including real quasicrystals. 


\section{Theory}

The Fibonacci chain is a model example of the 1D quasicrystal. This structure is mostly described in higher-dimensional (2D for the Fibonacci chain) space and then projected to lower-dimensional physical space [13-19]. The generalized projection method to produce quasilattices was first proposed by de Bruijn [13] and subsequently used by Kramer and Neri [14]. The projection operators written for the Cartesian coordinates $(x, y)$ are

$$
\begin{aligned}
& \boldsymbol{P}=[\cos \alpha, \sin \alpha], \\
& \boldsymbol{T}=[-\sin \alpha, \cos \alpha],
\end{aligned}
$$

where $\cot \alpha=\tau \equiv(1+\sqrt{5}) / 2 \approx 1.618 ; \boldsymbol{P}$ and $\boldsymbol{T}$ project the structure onto the real (parallel) and perpendicular (perp) subspaces, respectively. The real $\left(r_{\|}\right)$and perp $\left(r_{\perp}\right)$ space components of the $2 \mathrm{D}$ positional vector $\boldsymbol{R}(x, y)$ are given by

$$
\begin{aligned}
& r_{\|}=\boldsymbol{P} \cdot \boldsymbol{R}=x \cos \alpha+y \sin \alpha, \\
& r_{\perp}=\boldsymbol{T} \cdot \boldsymbol{R}=-x \sin \alpha+y \cos \alpha .
\end{aligned}
$$

To obtain the Fibonacci sequence of two bounds: large $L$ (with the length equal to $\tau$ ) and small $S$ (length equal to 1), the length of the 2D quadratic lattice should be $A=\sqrt{\tau+2}$. The corresponding reciprocal lattice is also quadratic with the length $K=2 \pi / A$. For a given $2 \mathrm{D}$ reciprocal vector

$$
\boldsymbol{K}=\left(k_{x}, k_{y}\right)=\frac{2 \pi}{A}\left(h_{x}, h_{y}\right)=\left[k_{\|}, k_{\perp}\right]
$$

where

$$
\begin{aligned}
& k_{\|}=\boldsymbol{P} \cdot \boldsymbol{K}=\frac{2 \pi}{\tau^{2}+1}\left(\tau h_{x}+h_{y}\right), \\
& k_{\perp}=\boldsymbol{T} \cdot \boldsymbol{K}=\frac{2 \pi}{\tau^{2}+1}\left(-h_{x}+\tau h_{y}\right) .
\end{aligned}
$$

The round brackets are for the Cartesian coordinates and the square brackets are for tilt rectangular coordinates along the real and perp spaces.

For the $2 \mathrm{D}$ diffraction peaks (at the reciprocal lattice points)

$$
\exp (\mathrm{i} \boldsymbol{K} \cdot \boldsymbol{R})=\exp \left[\mathrm{i}\left(k_{x} x+k_{y} y\right)\right]=\exp \left[\mathrm{i}\left(k_{\|} r_{\|}+k_{\perp} r_{\perp}\right)\right]=1,
$$

which leads to

$$
k_{\|}{ }^{r}+k_{\perp} r_{\perp}=2 \pi n, \quad n \in Z .
$$

In respect of the reference lattice constructed for a given scattering vector $k_{0}=k_{\|}$ $[9,11]$, the reduced atomic position is given by

$$
u \equiv r_{\|} \bmod (\lambda), \quad \text { where } \lambda=2 \pi / k_{0}
$$

Using (9) and (10) one can also write 


$$
r_{\perp}=-\frac{k_{\|}}{k_{\perp}} u+\frac{2 \pi n_{1}}{k_{\perp}}
$$

where $n_{1}$ is an integer.

The structure factor in the real space can then be written as

$$
F\left(k_{\|}\right)=\int_{0}^{\tau^{2}} P_{\perp}\left(r_{\perp}\right) \exp \left(-\mathrm{i} k_{\perp} r_{\perp}\right) \mathrm{d} r_{\perp}
$$

where $P_{\perp}\left(r_{\perp}\right)$ is the probability distribution of atomic positions in perp space (this distribution directly corresponds to the so-called atomic surface). The perp space distribution is bounded to the width of the strip equal to $\tau^{2}$. Using (11) one can write

$$
\begin{gathered}
F\left(k_{\|}\right)=\int_{0}^{-u_{0}}-\frac{k_{\|}}{k_{\perp}} P_{\perp}\left(-\frac{k_{\|}}{k_{\perp}} u+\frac{2 \pi n_{1}}{k_{\perp}}\right) \\
\times \exp \left[-\mathrm{i} k_{\perp}\left(-\frac{k_{\|}}{k_{\perp}} u+\frac{2 \pi n_{1}}{k_{\perp}}\right)\right] \mathrm{d} u
\end{gathered}
$$

where $u_{0} \equiv\left(k_{\perp} / k_{\|}\right) \tau^{2}$. The bounds of the integral have been reduced to the average unit cell only.

Knowing (10) one gets

$$
\begin{gathered}
\frac{k_{\|}}{k_{\perp}} P_{\perp}\left(-\frac{k_{\|}}{k_{\perp}} u+\frac{2 \pi n_{1}}{k_{\perp}}\right)=\frac{k_{\|}}{k_{\perp}} P_{\perp}\left[-\frac{k_{\|}}{k_{\perp}}\left(u+\lambda n_{1}\right)\right] \\
=\frac{k_{\|}}{k_{\perp}} P_{\perp}\left(-\frac{k_{\|}}{k_{\perp}} u\right) \equiv P_{\|}(u),
\end{gathered}
$$

which finally leads to

$$
F\left(k_{\|}\right)=\int_{-u_{0}}^{0} P_{\|}(u) \exp \left(i k_{\|} u\right) \mathrm{d} u .
$$

The above means that the structure factor can be calculated in real space using the statistical distribution, i.e. the average unit cell approach [9-11]. The average unit cell is defined by the probability distribution of atomic positions in respect of the corresponding reference lattice. Although the average unit cell is calculated for the particular scattering vector $k_{0}$, the obtained probability distribution describes the structure factor for all higher harmonics of the given wave vector (i.e. $k=$ $n k_{0}$ ). In $2 \mathrm{D}$ description this also means that formula (15) correctly describes the structure factor calculated along the line given by $k_{\perp} / k_{\|}=$const. It has also been pointed out [11] that the average unit cell is an oblique projection of the atomic surface onto physical space (formally there is also inversion which should be considered to compare the discussed distributions, however, it does not influence the diffraction pattern). The atomic surface is limited to $\left(0, \tau^{2}\right)$, with $P_{\perp}\left(r_{\perp}\right)=$ $1 / \tau^{2}$. The corresponding distribution in the physical space is bounded to $\left(-u_{0}, 0\right)$, with value $P(u)=1 / u_{0}$. 


\section{Modulation vector}

To describe all the diffraction peaks one needs the second wave vector, i.e. the modulation vector $q_{0}$. For the Fibonacci chain the modulation vector, describing the satellites reflections, is $\tau$ times shorter than the scattering vector of the main reflections $\left(k_{0}\right)$, i.e $q_{0}=k_{0} / \tau$. Using two reference lattices, the first one corresponding to $k_{0}$, and the second one - to $q_{0}$, one gets the probability distribution $P(u, v)$ [9-11], where $u$ and $v$ are the atomic distances to the corresponding reference lattices. Probability distribution $P(u, v)$ stands for the average unit cell in the statistical approach. The structure factor at the scattering vector given by $k=n k_{0}+m q_{0}, n$ and $m$ are integers, reads

$$
F(k)=\int_{-u_{1}}^{0} \int_{-v_{1}}^{0} P(u, v) \exp \left[-\mathrm{i}\left(n k_{0} u+m q_{0} v\right)\right] \mathrm{d} v \mathrm{~d} u .
$$

In $\mathrm{Eq}$. (16) the integral bounds are given by the boundaries of the average unit cell, i.e. $u_{1}=2 \pi / k_{0}$ and $v_{1}=2 \pi / q_{0}$. In many cases, however, like in the discussed one of the Fibonacci chain, the distribution is additionally bounded to the range of $u_{0}<u_{1}$ and $v_{0}<v_{1}$. An arbitrary shift of the obtained distribution does not influence the diffraction pattern.

It has already been noticed $[9,10]$ that for $\tau$ times shorter scattering vector in the real space, its perp space components become $\tau$ times longer and finally the bound $v_{0}$, which is proportional to the ratio of the perp space to the real space components of the integral, should be $\left(-\tau^{2}\right)$ times larger than $u_{0}$, i.e. $v_{0}=-u_{0} \tau^{2}$. This leads to the following:

$$
F(k)=\int_{-u_{0}}^{0}\left\{\int_{u_{0} \tau^{2}}^{0} P(u, v) \exp \left[-\mathrm{i}\left(n k_{0} u+m q_{0} v\right)\right] \mathrm{d} v\right\} \mathrm{d} u,
$$

where $k_{\|} \equiv k_{0}$ and $q_{0}=k_{0} / \tau$. When the distribution is shifted by $a_{0}$ the expression for the structure factor is modified by a phase factor as following:

$$
\begin{aligned}
F(k) & =\int_{-u_{0}+a_{0}}^{a_{0}} \int_{u_{0} \tau^{2}+a_{0}}^{a_{0}} P(u, v) \exp \left[-\mathrm{i}\left(n k_{0} u+m q_{0} v\right)\right] \mathrm{d} v \mathrm{~d} u \\
& =\exp \left(-\mathrm{i} k a_{0}\right) \int_{-u_{0}}^{0} \int_{u_{0} \tau^{2}}^{0} P(u, v) \exp \left[-\mathrm{i} k_{0}\left(n u+\frac{m}{\tau} v\right)\right] \mathrm{d} v \mathrm{~d} u .
\end{aligned}
$$

Such a phase factor does not influence the diffraction pattern but it is crucial for the decorated structures. Probability distribution is non-zero only along the line given by the expression $v=-\tau^{2} u+c$, which leads to

$$
\begin{aligned}
F(k) & =\exp \left(-\mathrm{i} k a_{0}\right) \int_{-u_{0}}^{0}\left(\int_{u_{0} \tau^{2}}^{0} P(u, v(u)) \mathrm{d} v\right) \\
& \times \exp \left\{-\mathrm{i} k_{0}\left[n u+\frac{m}{\tau}\left(-\tau^{2} u+c\right)\right]\right\} \mathrm{d} u \\
& =\exp \left[-\mathrm{i}\left(k a_{0}+c_{1}\right)\right] \int_{-u_{0}}^{0} P(u) \exp \left[-\mathrm{i} k_{0}(n-\tau m) u\right] \mathrm{d} u
\end{aligned}
$$


Knowing that

$$
k=n k_{0}+m q_{0}=k_{0}(n+m / \tau)=k_{0}(n-\tau m)+k_{0} m \sqrt{5},
$$

one can also write

$$
F(k)=\exp \left[-\mathrm{i}\left(k a_{0}+c_{1}\right)\right] \int_{-u_{0}}^{0} P(u) \exp \left(-\mathrm{i} k_{m} u\right) \mathrm{d} u,
$$

where $c_{1}$ is a constant, $k_{m} \equiv k-k_{0} m \sqrt{5}$ is the reduced scattering vector for the group of $m$-order satellites. Supposing that $k$ is a continuous variable, $|F(k)|^{2}$ describes a curve, called as envelope function, connecting peaks' intensities of the appropriate group of satellites. For $m=0$ this envelope connects the periodic set of main reflections. Knowing only the distribution $P(u)$, which for the Fibonacci chain has a rectangular shape, i.e. $P(u)=1 / u_{0}$ for $-u_{0}<u \leq 0$, one can fully describe the corresponding diffraction pattern.

\section{Small and large bounds}

The question, which has to be answered, is the following: which part of the probability distribution has to be connected with short and large bounds respectively? It is easy to solve this problem in 2D. The length of the atomic surface is equal to $\tau^{2}$. All the horizontal sides of the quadratic structures belonging to the projected strip give large bounds; the vertical sides bring to the short bounds of the Fibonacci chain. Let us consider the short bound: there should be two points separated by $\tau$ in perp space belonging to the projected strip of the full width equal to $\tau^{2}$ (the width of the atomic surface). This means that the following equation holds for the perp space position of atoms decorating the small bound: $s_{\text {perp }}+\tau<\tau^{2}$, which brings to the solution $s_{\text {perp }} \in\langle 0,1)$. Similarly for the atoms decorating the large bound one gets $l_{\text {perp }} \in\left\langle 1, \tau^{2}\right)$. When projected to the real space (oblique projection perpendicular to the chosen wave vector $\left.K=\left(k_{\|}, k_{\perp}\right)\right)$ one can conclude: for $u \in\left(0,-u_{\mathrm{s}}\right)$ the distribution describes the short bounds only, and for $u \in\left(-u_{\mathrm{s}},-u_{0}\right)$ - the large bounds of the Fibonacci chain, where $u_{\mathrm{s}}=k_{\perp} / k_{\|}$and $u_{0}=\left(k_{\perp} / k_{\|}\right) \tau^{2}$. The $u_{\mathrm{s}}$ position divides the rectangular statistical distribution of the Fibonacci chain into two distributions: the first one connected with the $S$ bound, the second one - with the $L$ bound. Then one can write

$$
F(k)=F_{S}(k)+F_{L}(k),
$$

where

$$
\begin{aligned}
& F_{S}(k)=\int_{-u_{\mathrm{s}}}^{0} P(u) \exp \left(-\mathrm{i} k_{m} u\right) \mathrm{d} u \\
& F_{L}(k)=\int_{-u_{0}}^{-u_{\mathrm{s}}} P(u) \exp \left(-\mathrm{i} k_{m} u\right) \mathrm{d} u
\end{aligned}
$$




\section{Decoration}

Let us suppose that the small bound of the Fibonacci chain is decorated by $n_{S}$ atoms at relative positions given by $\left\{s_{j}\right\}, j=1, n_{S}$. Similarly the large bounds are decorated by $n_{L}$ atoms and the relative positions of decorating atoms are: $\left\{l_{j}\right\}, j=1, n_{L}$. Using (21) and (22) one can write the structure factor for decorated Fibonacci chain as

$$
F(k)=F_{S}(k) \sum_{j=1}^{n_{S}} \exp \left(-\mathrm{i} k s_{j}\right)+F_{L}(k) \sum_{j=1}^{n_{L}} \exp \left(-\mathrm{i} k l_{j}\right) .
$$

Using (23) and (24) in (25) one finally gets

$$
\begin{aligned}
F(k) & =\frac{\tau}{n_{L} \tau+n_{S}}\left\{\frac{\sin (w)}{w} \sum_{j=1}^{n_{L}} \exp \left[\mathrm{i}\left(-k l_{j}+k_{m} u_{0} \frac{3-\tau}{2}\right)\right]\right. \\
& \left.+\frac{1}{\tau} \frac{\sin (w / \tau)}{w / \tau} \sum_{j=1}^{n_{\mathrm{s}}} \exp \left[\mathrm{i}\left(-k s_{j}+k_{m} u_{0} \frac{2-\tau}{2}\right)\right]\right\}
\end{aligned}
$$

where

$$
w=\frac{k_{m} u_{0}}{2 \tau}=\frac{\left(k-m k_{0} \sqrt{5}\right) u_{0}}{2 \tau} .
$$

The above equations fully describe the structure factor of arbitrary decorated Fibonacci chain. In case of decoration by different atoms, the corresponding atomic form factors should be added before the exponential functions. Equation (26) can be essentially reduced for the particular decoration. The simplest decorations are discussed below in two examples: the first one is for the single atomic decoration in the centres of each bounds, the second one is some other decoration which keeps the Fibonacci ratio of large to small bound.

\section{Example 1}

The simplest decoration is the following: let us suppose that the "atoms" are placed at the centres of each short and large bounds. In such a case there are only two positions: $s_{1}=0.5$ and $l_{1}=\tau / 2 \approx 0.809$. The mean distance between the atoms is

$$
a_{0}=1+\frac{1}{\tau^{2}} \approx 1.319
$$

i.e. the same as for non-decorated Fibonacci chain. The corresponding wave vectors are

$$
\begin{aligned}
& k_{\|} \equiv k_{0}=\frac{2 \pi}{a_{0}}=\frac{2 \pi}{\tau^{2}+1}(\tau+1) \approx 4.547 \Rightarrow h_{x}=1, h_{y}=1, \\
& k_{\perp}(1,1)=\frac{2 \pi}{\tau^{2}+1}(-1+\tau) \approx 1.073
\end{aligned}
$$


and

$$
q_{0}=\frac{k_{0}}{\tau} \approx 2.810
$$

with the ratio

$$
\frac{k_{\perp}}{k_{\|}}=\frac{1}{\tau^{3}} \approx 0.236
$$

The obtained $P(u, v)$ distribution is shown in Fig. 1a and its projection to $u$-parameter space - in Fig. 1b. For this particular decoration the structure fac-
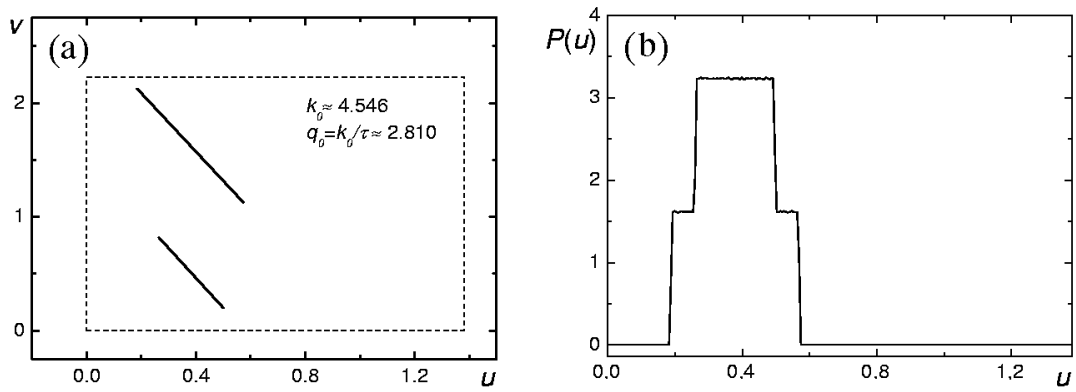

Fig. 1. Probability distribution of distances for the Fibonacci chain with the atoms placed at the centres of each bound (example 1). (a) In a double parameter space the distribution is non-zero only along the certain lines marked in the figure together with the shape of the average unit cell (dashed rectangle). (b) Projection of the distribution onto $u$-parameter space. Calculations were performed for the scattering vectors $k_{0} \approx 4.55$ and $q_{0}=k_{0} / \tau \approx 2.81$ and number of atoms of about 35 thousand.

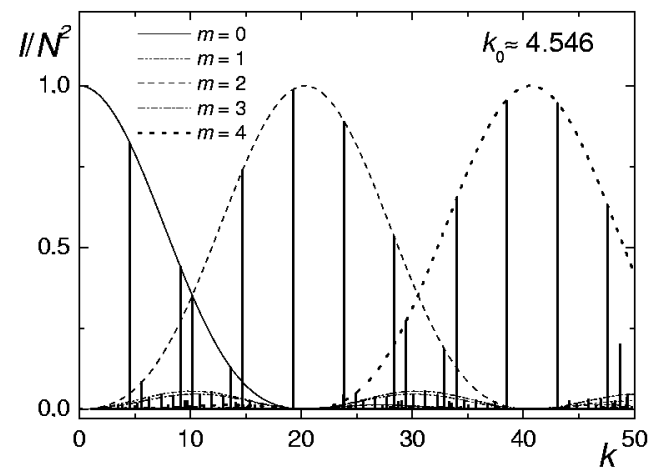

Fig. 2. Diffraction pattern of decorated Fibonacci chain when the atom is placed at the centre of each bounds. All the diffraction peaks are perfectly described by the envelope functions calculated from the derived Eq. (32). 
tor $(26)$ can be written as

$$
\begin{aligned}
F(k) & =\frac{1}{\tau}\left\{\frac{\sin w}{w} \exp \left[\mathrm{i}\left(k(\tau-2)-m k_{0} \sqrt{5} \frac{3 \tau-4}{2}\right)\right]\right. \\
& \left.\left.+\frac{1}{\tau} \frac{\sin (w / \tau)}{w / \tau} \exp \left[\mathrm{i}(k \tau-2)-m k_{0} \sqrt{5} \frac{2 \tau-3}{2}\right)\right]\right\} .
\end{aligned}
$$

Equation (33) was used for the calculation of the envelope functions for the main reflections $(m=0)$ and the $m$-th order satellites respectively, as it is shown in Fig. 2. The diffraction pattern is essentially different from the one calculated for the Fibonacci chain, however, Eq. (33) fully describes all the diffraction peaks for the discussed structure, irrespectively of their intensity.

\section{Example 2}

The second example is for the well known "Fibonacci-type" decoration $[10,20,21]$ given by: $L \rightarrow l l s l$ and $S \rightarrow$ sls. The small letters in the substitution means that the lengths of the new bounds are $(2+\tau)$ times smaller than the original ones. This substitution does not change the ratio of numbers of large to small bounds, which is given by $\tau$. The decorations of the large and small bounds (atomic positions) of the Fibonacci chain are the following:

- for the large bound

$$
\left(0, \frac{\tau}{2+\tau}, \frac{2 \tau}{2+\tau}, \frac{2 \tau+1}{2+\tau}\right) \approx(0,0.447,0.894,1.171)
$$

- for the small bound

$$
\left(0, \frac{1}{2+\tau}, \frac{1+\tau}{2+\tau}\right) \approx(0,0.276,0.723) \text {. }
$$

For this decoration one can easily explain the way in which the average unit cell is constructed. Let us start from the wave vectors equal to $k_{0} \approx 4.55$ and $q_{0} \approx 2.81$, as it is given by (29) and (31). The average unit cell in the parameter space $(u, v)$ is marked as dashed rectangle in Fig. 3a. Three atoms decorate the small $S$ bounds and for the first bound their positions were marked as closed circles in the figures. They are lying along the line given by the expression: $v=u$ and are placed at the positions given by the decoration (35). The other atoms decorating the $S$ bounds give the distributions which are non-zero only along the lines given by the expressions

$$
v=-\tau^{2} u+c
$$

with the length of corresponding bounds equal to

$$
a_{S}=\frac{1}{\tau^{3}} \sqrt{\tau^{4}+1} \approx 0.66
$$



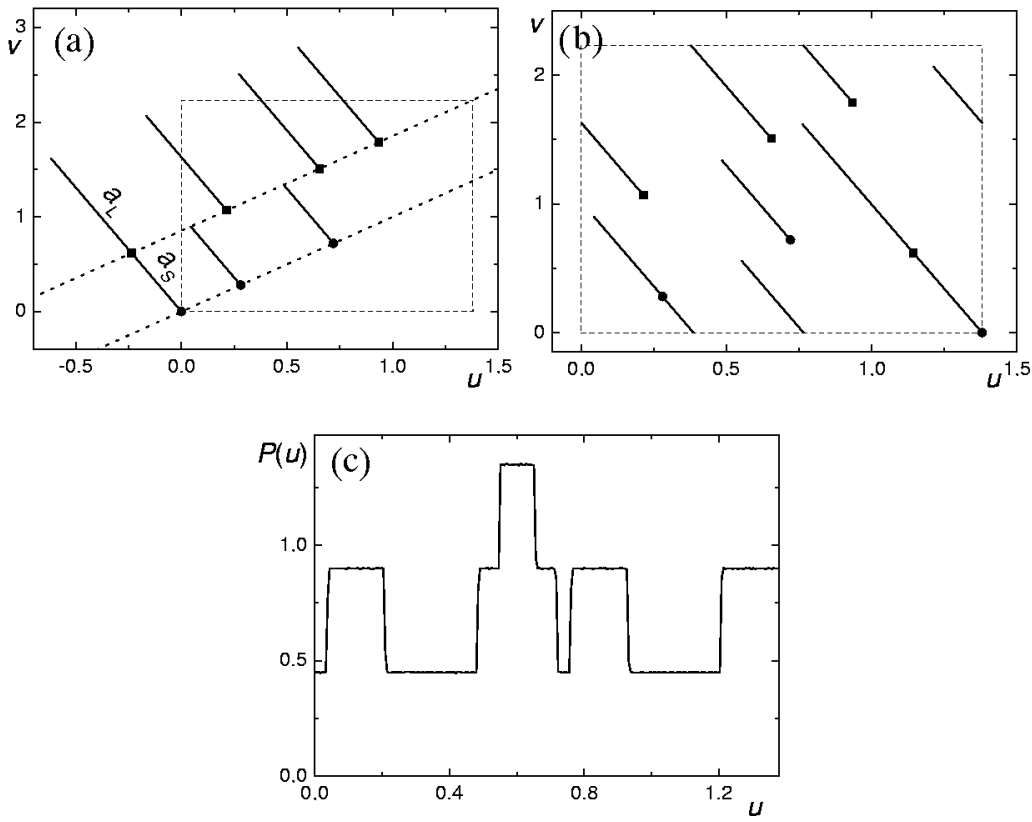

Fig. 3. Probability distribution obtained for the decorated structure with the following decoration: $L \rightarrow l l s l$ and $S \rightarrow$ sls. Four atoms (black squares) decorate the first $L$ bound and three atoms (filled circles) decorate the first $S$ bound. The distribution is non-zero only along particular directions marked in the figure. For the chosen wave vectors: $k_{0} \approx 4.55$ and $q_{0}=k_{0} / \tau \approx 2.81$, boundaries of the corresponding average unit cell were also marked in the figure by dashed lines. The extended average unit cell (shown in part (a)), when reduced to the single unit cell is presented in part (b). The projected onto $u$-space probability distribution is similar to the one shown in part (c). However, this part (c) was calculated as a statistical distribution of about 120 thousand atoms.

as it is marked in Fig. 3a. The $L$ bounds are decorated by four atoms indicated for the first bound as squares in Fig. 3a. They are also lying along the single line given by the expression $v=u+$ const. The appropriate statistical distribution smears out the atom into a bound of the length $a_{L}$, with the ratio $a_{L} / a_{S}=\tau$, as it is marked in the figures. Using the above procedure one obtains all the distributions in a very clear and simply way, however many of them are out of bounds of the average unit cell. After reduction to a single unit cell the distribution has been shown in Fig. 3b. Its projection onto $u$-parameter space has been also plotted in Fig. 3c. Fourier transform of the distribution functions from Fig. 3 brings to the envelope functions which connect intensities of each group of reflections, i.e. the main peaks first-order satellites and so on. Some of them were plotted in Fig. 4. In this figure, however, the very weak peaks, with the relative intensities between 0.0035 and 0.06 , were shown. One can easily see that all the diffraction peaks are perfectly 


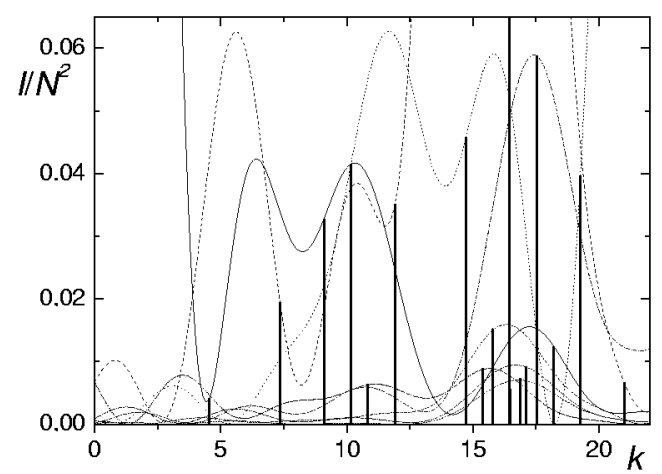

Fig. 4. Diffraction pattern of the decorated structure with the following decoration: $L \rightarrow l l s l$ and $S \rightarrow$ sls. In the figure the diffraction peaks have been connected by the envelope function calculated according to $(26)$ for the $k_{0} \approx 4.55$ and different $m$. Only very weak peaks, wich relative intensities between 0.0035 and 0.06 were shown.
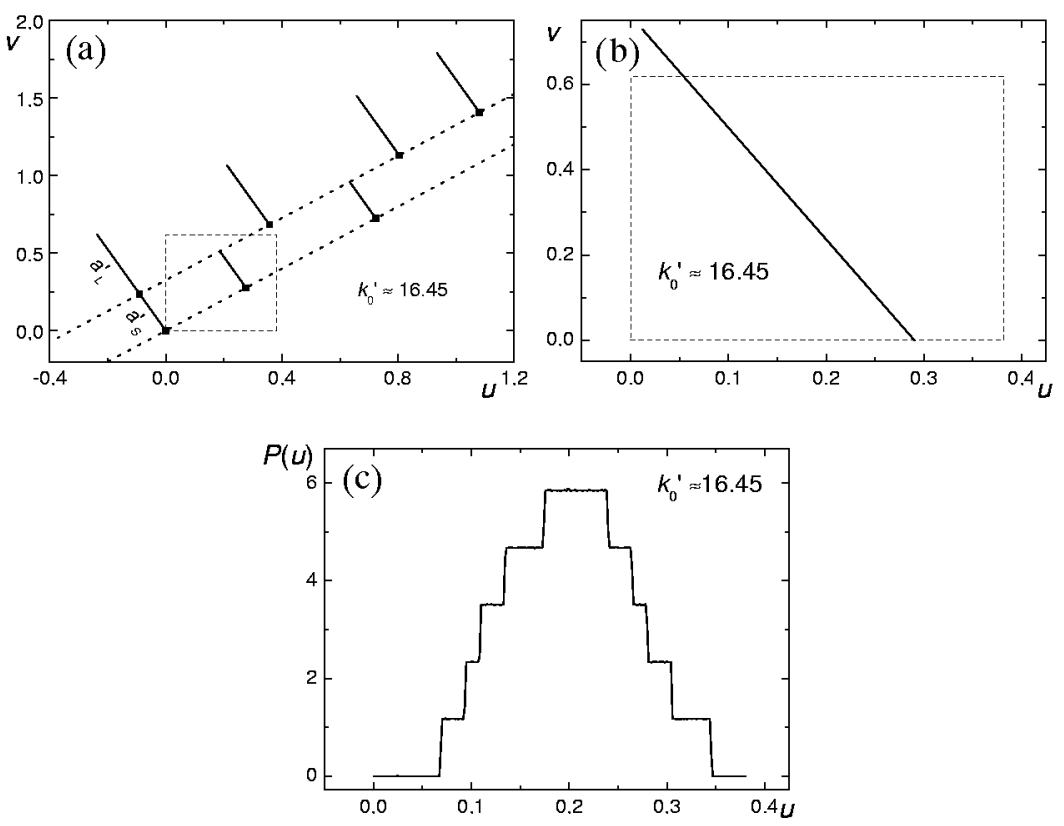

Fig. 5. Different average unit cells calculated for the decoration $L \rightarrow l l s l$ and $S \rightarrow s l s$ and the wave vector $k_{0}^{\prime}=k_{0}(\tau+2) \approx 16.45$ : (a) extended unit cell, (b) single unit cell, (c) projected unit cell (for about 120 thousand of atoms).

described in the average unit cell approach, which is the reason that there are no approximations in the used approach. However, such rather complicated envelope curves can be essentially reduced for the scattering vector $(2+\tau)$ times longer, i.e. 


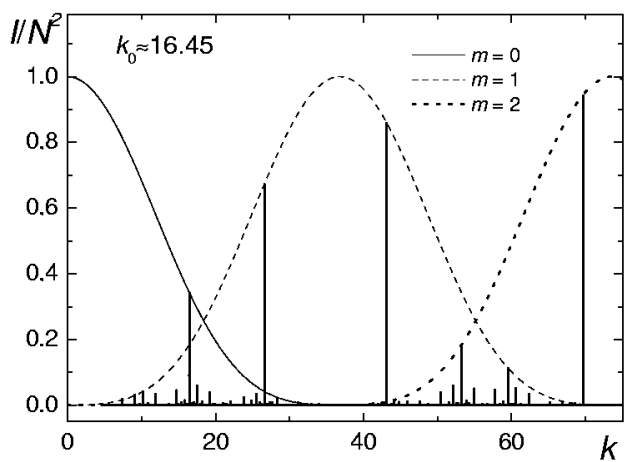

Fig. 6. The diffraction pattern for the decorated Fibonacci chain: $L \rightarrow l l s l$ and $S \rightarrow s l s$. The most intensive peaks were connected by the envelope function calculated from the distribution shown in Fig. 5c.

$k_{0}^{\prime}=k_{0}(\tau+2) \approx 16.45$. This also leads to $(2+\tau)$ times shorter distances and, for example, the new $a_{S}^{\prime}=a_{S} /(2+\tau) \approx 0.18$. The average unit cell for the new wave vector can be constructed in the very similar way as before (Figs. 5a, b and c). For this particular decoration the obtained probability distribution in the unit cell is non-zero only along the single line as it is shown in Fig. 5b. In the discussed case one obtains a very simple envelope function which perfectly describes only particular subset of diffraction peaks (Fig. 6).

\section{Conclusions}

In this paper the structure factor was derived in physical space for arbitrary decorated Fibonacci chain. Using statistical approach (average unit cell approach) one gets the formula (26) which describes the structure factor for each group of peaks indexed by $m$, i.e. the main reflections $(m=0)$, the first-order satellites $(m=1)$, the second-order satellites $(m=2)$ and so on. The presented approach is parallel to the higher-dimensional analysis which is commonly used. To see this parallelism the real-space $\left(k_{\|}\right)$and the perp-space $\left(k_{\perp}\right)$ components were also used for the description of the scattering vector, exchangeable to the average unit cell description given by the main scattering vector $\left(k_{0}\right)$ and the modulation vector $\left(q_{0}\right)$. Using Eq. (26) the problem of the structure determination is reduced to the refinement, from the diffraction pattern, of the atomic positions in the two bounds of the Fibonacci chain. The whole procedure is limited to the physical space only and the number of refining parameter is only one for each atom, i.e. to the position of each atom in the particular $L$ or $S$ bounds. In that sense the refining is no more complicated than for the 1D crystals and the standard methods can be applied. The only difference is the indexing problem and grouping the reflections into different classes: the main reflections and the satellites of a given order. The presented 
approach can be easily applied to the 2D and 3D structures. The calculations are in progress and the results will be discussed separately.

\section{References}

[1] D. Shechtman, I. Blech, D. Gratias, J.W. Cahn, Phys. Rev. Lett. 53, 1951 (1994).

[2] P. Bak, Phys. Rev. Lett. 56, 861 (1986).

[3] T. Haibach, W. Steurer, Acta Crystallogr. Sec. A: Found. Crystallogr. 52, 277 (1996).

[4] W. Steurer, T. Haibach, in: Physical Properties of Quasicrystals, Ed. Z.U. Stadnik, Springer, Berlin 1999, p. 51.

[5] M. de Boissieu, Z. Kristall. 215, 597 (2000).

[6] H. Takakura, M. Shiono, T.J. Sato, A. Yamamoto, A.P. Tsai, Phys. Rev. Lett. 86, 236 (2001).

[7] E. Abe, S.J. Pennycook, A.P. Tsai, Nature 421, 347 (2003).

[8] E. Abe, J.Q. Guo, A.P. Tsai, Philos. Mag. Lett. 81, 563 (2001).

[9] J. Wolny, Philos. Mag. A 77, 395 (1998).

[10] J. Wolny, L. Pytlik, Acta Crystallogr. Sec. A: Found. Crystallogr. 56, 11 (2000).

[11] J. Wolny, B. Kozakowski, P. Repetowicz, J. Alloys Comp. 342, 198 (2002).

[12] A. Cervellino, W. Steurer, Acta Crystallogr. Sec. A: Found. Crystallogr. 58, 180 (2002).

[13] N.G. de Bruijn, Proc. K. Ned. Akad. Wet. Ser. A 43, 39 (1981).

[14] P. Kramer, R. Neri, Acta Crystallogr. A 40, 580 (1984).

[15] M. Duneau, A. Katz, Phys. Rev. Lett. 54, 2688 (1985).

[16] P. Bak, Scr. Metall. 20, 1199 (1986).

[17] V. Elser, Acta Crystallogr. A 42, 36 (1986).

[18] M.V. Jaric, Phys. Rev. B 34, 4685 (1986).

[19] T. Janssen, Phys. Rep. 168, 55 (1988).

[20] F. Lancon, L. Billard, S. Burkov, M. de Boissieu, J. Phys. (France) 4, 283 (1994).

[21] A. Cervellino, T. Haibach, W. Steurer, Phys. Rev. B 57, 11223 (1998). 